\title{
Medición de la comorbilidad depresiva en drogodependientes: Validez y eficacia diagnóstica del Inventario de Depresión de Beck (BDI-II) en dominicanos con múltiple consumo de sustancias
}

\author{
Zoilo E. García-Batista ${ }^{1}$, Kiero Guerra-Peña ${ }^{1}$, Antonio Cano-Vindel ${ }^{2}$, Luisa M. Cantisano-Guzmán ${ }^{1}$, \\ Pablo E. Flores-Kanter ${ }^{3}$ y Leonardo A. Medrano ${ }^{1,3}$

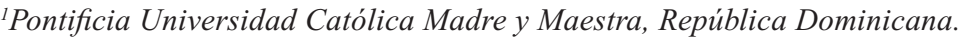 \\ ${ }^{2}$ Universidad Complutense de Madrid, Madrid, España. \\ ${ }^{3}$ Universidad Siglo 21, Córdoba, Argentina.
}

\begin{abstract}
Depressive comorbidity measurement in drug dependents: Validity and diagnostic efficiency of Beck's Depression Inventory (BDI-II) in Dominicans with multiple substance consumption. The present study proposed two main objectives, a) evaluate the validity of BDI-II for the identification of comorbid depressive symptoms in people with multiple substance abuse (i.e. Known-Groups Validity); and b) verify the diagnostic efficacy of the inventory for the distinction between general population and clinical drug dependents. From a two-stage sampling, a total of 116 Dominicans was selected, of whom 50\% were drug-dependent. Analysis of differences between groups and analysis of COR curves were applied. The results show statistically significant and large effect size differences in depression levels in favor of the substance abuse group. In addition, COR curve analyses indicate that depression scores derived from BDI-II have adequate diagnostic performance indices. In short, evidence is provided in favor of the appropriateness and validity of the BDI-II to be applied in a population of drug dependents.
\end{abstract}

Keywords: Drug dependents; depression; BDI-II; comorbidity; known-groups validity; COR curves; Dominican Republic.

Resumen: El presente estudio se propuso como objetivos, a) evaluar la validez del BDI-II para la identificación de sintomatología depresiva comórbida en personas con múltiple abuso de sustancias (i.e. Known-Groups Validity); y b) verificar la eficacia diagnóstica del inventario para la distinción entre población general y drogodependientes clínicos. A partir de un muestreo bietapico, se seleccionó un total de 116 dominicanos de los cuales el 50\% era drogodependiente. Se aplicaron análisis de diferencias entre grupos y análisis de curvas COR. Los resultados muestran diferencias estadísticamente significativas, y de tamaño del efecto grande, en los niveles de depresión en favor del grupo de personas con problemática de abuso de sustancias. Además, los análisis de curvas COR indican que los puntajes de depresión derivados del BDI-II presentan índices de rendimiento diagnóstico adecuados. En suma, se aporta evidencia a favor de la adecuación y validez del BDI-II para ser aplicado en población de drogodependientes.

Palabras clave: Drogodependencia; depresión; BDI-II; comorbilidad; known-groups validity; curvas COR; República Dominicana.

Recibido: 25 de marzo 2019; aceptado: 12 de noviembre 2019 Correspondencia: Zoilo Emilio García-Batista, Pontificia Universidad Católica Madre y Maestra, Autopista Duarte Km 1 1/2, Santiago de los Caballeros, República Dominicana. Correo-e: zgarcia@pucmm.edu.do.

Agradecimientos: Esta investigación contó con el apoyo del Fondo Nacional de Innovación y Desarrollo Científico y Tecnológico (FONDOCYT) de la República Dominicana. Los autores desean agradecer sinceramente a MESCyT y PUCMM por su sólido apoyo financiero, administrativo y técnico.

\section{Introducción}

La depresión es un desorden emocional de alta prevalencia e impacto nivel mundial. En 2015, se observó que 322 millones de personas alrededor del mundo (4.4\% de la población) sufrían de depresión, siendo declarada como la causa singular que provoca más incapacidad globalmente. En América, 48.16 millones de personas (15\% del total de los 322 millones) padecían la enfermedad, afectando a 
464,164 dominicanos (4.7\% de los habitantes del país). Para estas personas, la depresión puede traer consecuencias significativamente negativas en el desempeño en todos los ámbitos en los que se desenvuelvan, mermando con ello su calidad de vida y su bienestar psicológico general (Organización Mundial de la Salud, 2018).

Entre las consecuencias negativas derivables de la depresión se encontraría la drogodependencia o el consumo abusivo de sustancia. Si bien los mecanismos neurobiológicos y cognitivos que explican esta comorbilidad aún están siendo estudiados, existe mucha evidencia de la fuerte asociación entre la depresión y diversas problemáticas de consumo de drogas (Beaulieu, et al., 2018; Crnić, Todorović, Marković, Kastratović, y Timotijević, 2016; Gajewski, Turecki, y Robison, 2016; García-García, Luque, Santos Ruiz, y Tabernero, 2017; Gómez-Coronado, et al., 2018; Hammond, Lai, Wright, y Treisman, 2016; Khalid y Kausar, 2016; Hodgson, et al., 2017; Lai, Cleary, Sitharthan, y Hunt, 2015; Lister, Ledgerwood, Lundahl, y Greenwald, 2015; Rao, 2006). Una de las principales hipótesis que se han propuesto para la explicación de esta comorbilidad entre depresión y abuso de sustancia, es la hipótesis de automedicación (self-medication hypothesis; Gómez-Coronado, et al., 2018). Se afirma que los componentes cognitivos de la depresión, como ser los pensamientos de fracaso, o de disconformidad con uno mismo, amplificarían la afectividad negativa, siendo el consumo una forma conductual de regulación emocional que buscaría aminorar dicha afectividad (Obasi, Brooks, y Cavanagh, 2016). Esta hipótesis es coincidente con el Modelo de Cascada Emocional (Emotional Cascade Model; Selby, Anestis, y Joiner, 2008; Selby, Franklin, Carson-Wong, y Rizvi, 2013) así como los modelos transdiagnósticos de la regulación emocional (Sloan, et a., 2017), en donde se indica que ciertas respuestas o contenidos cognitivas generan un incremento de la afectividad negativa, a partir de lo cual personas que no posen otros recursos más apropiados recurren a modos de afrontamiento maladaptativos, como ser el consumo de sustancia, con el objeto de disminuir este estado afectivo negativo.

Sumado a lo anterior, se señala una segunda vía a partir de la cual la depresión se asociaría al consumo de sustancia, más vinculada con la afección a nivel somático que pueden provocar los periodos de intoxicación, desintoxicación y/o abstinencia (Johnson, Neal, Brems, y Fisher, 2006; Skule, et al., 2014). Entre los procesos involucrados, se hace hincapié en la hipótesis del uso de drogas para aumentar el placer. En relación con esto, algunos autores han encontrado que la búsqueda de placer es una de las principales causas por las que las personas recurren al consumo de sustancias psicoactivas (Kennet, Mathews, y Snoek, 2013; Pordanjani, et al., 2018; Webb, Ashton, y
Kamali, 1996). Desde un plano neurobiológico, el estado de ánimo depresivo o disfórico estaría reflejando una alteración en los sistemas cerebrales de motivación y recompensa (Gómez-Coronado, et al., 2018; Rao, 2006).

Más allá de estas consideraciones teóricas, y a pesar de la amplia evidencia de la comorbilidad existente entre depresión y problemáticas relacionadas al consumo de sustancias, existen aún controversias acerca de los instrumentos aplicados para la identificación de la sintomatología depresiva en drogodependientes. En este contexto se postula la necesidad de utilizar instrumentos psicométricos válidos, rápidos y eficientes para identificar depresión en personas con problema de abuso de sustancias (Johnson, et al., 2006; Seignourel, Green, y Schmitz, 2008). El Inventario de Depresión de Beck-II (BDI-II), uno de los instrumentos más aplicados e investigado a nivel internacional (Sanz, Gutiérrez, Gesteira, y García-Vera, 2014), ha sido también identificado como un instrumento idóneo para su utilización en el estudio de la comorbilidad entre depresión y abuso de sustancias (Skule, et al., 2014). Sin embargo, la evidencia acerca de la validez del BDI-II para los fines descritos es aún escasa.

A la fecha hemos podido identificar solo tres antecedentes en donde se han analizado las propiedades psicométricas del BDI-II considerando muestras clínicas de personas con problemática de consumo de sustancias. En el estudio más reciente, Skule, et al., (2014) indagaron la estructura factorial del inventario en participantes con depresión clínica, con y sin abuso de consumo de alcohol. Se encuentra evidencia a favor de una estructura de tres factores (i.e. Cognitiva, Afectiva, y Somática), y se verifica la invarianza factorial entre los grupos. En cuanto a los niveles de depresión, se encuentra una diferencia de moderada magnitud en favor del grupo con comorbilidad de abuso de alcohol. Por su parte, Seignourel, et al., (2008) evaluaron la estructura factorial en personas drogodependientes (i.e. dependencia al alcohol, cocaína, y opioides), encontrando evidencia también en favor de esta estructura trifactorial. Además, los autores encuentran que las puntuaciones del BDI-II tienen una adecuada eficacia diagnóstica para diferenciar entre drogodependientes depresivos y no depresivos. Similar a los anteriores hallazgos, Johnson, et al. (2006) encontró evidencia en favor de esta estructura de tres factores, tomando una muestra de individuos con policonsumo y uso de drogas callejeras inyectables (i.e. morfina, cocaína, y anfetaminas). Si bien los antecedentes mencionados aportan evidencia a favor de la validez de las puntuaciones del BDI-II al aplicarse personas con problemática de abuso de sustancias, hay una serie de aspectos que no han sido indagados que permitirían la obtención de mayor evidencia en esta área investigación. 
Tabla 1. Datos sociodemográficos en función de los grupos contrastados

\begin{tabular}{lcccc}
\hline & \multicolumn{2}{c}{ Muestras } & & \\
\cline { 2 - 3 } & Población general & Drogodependientes & & \\
\hline Edad & 34.81 & 34.48 & $t=.14$ & .88 \\
$\quad$ \% Sexo masculino & 84.50 & 84.50 & $\chi^{2}=.01$ & .99 \\
Estado Civil & & & $<.01$ \\
$\quad$ \% solteros & 65.50 & 80.00 & $\chi^{2}=12.10$ & \\
Escolaridad & & & $<.01$ \\
$\quad \%$ ninguna & 3.40 & 21.40 & $\chi^{2}=56.40$ & \\
\hline
\end{tabular}

Entre los aspectos sobre los cuales no se ha aportado evidencia suficiente se encuentra la evaluación de la validez discriminativa (i.e. Known-Groups Validity) de las puntuaciones del BDI-II entre personas con múltiple abuso de sustancias y población general. En otras palabras, todavía no se ha indagado si los puntajes obtenidos por este inventario son consistentes entre personas con consumo problemático de distintas sustancias (e.g. cocaína vs nicotina), y no se ha considerado el abuso de algunas sustancias relevantes (i.e. crack y nicotina). Tampoco se ha indagado hasta la fecha la eficacia diagnóstica del BDI-II, tanto en su dimensión general como considerando sus subdimensiones, respecto a la distinción entre población general y drogodependientes clínicos.

Dado el panorama actual, el presente estudio se propone los siguientes objetivos: a) verificar la validez del BDI-II para identificar la comorbilidad depresiva en población de drogodependientes, considerando el consumo problemático de distintas sustancias (i.e. marihuana, cocaína, crack, alcohol, y nicotina), algunas hasta la fecha no consideradas; y b) verificar la eficacia diagnóstica, respecto a la distinción entre población general y drogodependientes clínicos, del BDI-II. El presente trabajo no solo pretende generar evidencia en relación a los objetivos antes mencionados, sino además verificar si los resultados obtenidos en contextos de países desarrollados son generalizables a poblaciones de menor desarrollo (Khon, et al., 2018; Rad, Martingano, y Ginges, 2018), en este caso República Dominicana.

\section{Metodología}

\section{Participantes}

En este estudio participaron 116 dominicanos, seleccionados a partir de un muestreo de dos etapas. En la primera etapa se seleccionó a los participantes que conformarían el grupo clínico de drogodependientes. Este grupo quedó conformado por 58 internos (i.e. personas que padecen drogo- dependencia y permanecen internados para su tratamiento), pertenecientes a una institución de rehabilitación para drogodependientes de República Dominicana, Hogar Crea Dominicana. Conformado el primer grupo, la segunda etapa de muestreo consistió en seleccionar a los participantes de la población general, manteniendo la misma cuota en cuanto a sexo y grupos etarios, así como la misma cantidad de personas, respecto al grupo clínico. De esta forma, se buscó principalmente que los grupos quedaran equilibrados en sus respectivas variables sociodemográficas. Cada uno de los grupos quedó finalmente constituido por 49 hombres (84.48\%) y 9 mujeres (15.52\%). Además, la media de edad general de los participantes fue de $34.79(\mathrm{DE}=12.44)$ años, siendo la del grupo de población general de $34.81(\mathrm{DE}=$ 13.02), mientras que la del grupo clínico de 34.77 ( $\mathrm{DE}=$ 11.93) años. En la Tabla 1 se da mayor detalle de los datos sociodemográficos en función de los grupos.

En la Tabla 2 se describen los datos de consumo del grupo clínico.

Tabla 2. Consumo de sustancias en el grupo de personas drogodependientes

\begin{tabular}{lcc}
\hline & \multicolumn{2}{c}{ Consumo } \\
\cline { 2 - 3 } & $n$ & $\%$ \\
\hline Marihuana & 37 & 63.8 \\
Cocaína & 43 & 74.1 \\
Crack & 20 & 34.5 \\
Alcohol & 20 & 34.5 \\
Nicotina & 14 & 24.1 \\
\hline
\end{tabular}

\section{Instrumentos}

Inventario de Depresión de Beck-II (BDI-II; Beck, Steer, y Brown, 1996). Permite evaluar la presencia y la severidad de sintomatología depresiva, basándose en los síntomas propuestos por el Manual Diagnóstico y Estadístico de los Desórdenes Mentales (DSM-IV; American 
Psychiatric Association, 2000). El mismo es un autoinforme conformado por 21 ítems, los cuales se responden de acuerdo a una escala de 0 a 3 , en la cual 3 representa la opción de mayor gravedad del síntoma depresivo medido. Este test ha demostrado niveles adecuados de confiabilidad y validez. En el estudio de García-Batista, Guerra-Peña, Cano-Vindel, Herrera-Martínez, y Medrano (2018), se encontró que el instrumento era válido y confiable para la evaluación de la depresión en personas de la República Dominicana. Dentro de los modelos factoriales que se analizaron en el citado estudio, el modelo bifactor obtuvo los mejores índices de ajustes. En el modelo bifactor se evidencia una dimensión general de severidad depresiva, así como tres factores específicos ortogonales: cognitivo, somático y afectivo. En el presente estudio se encuentra evidencia de fiabilidad similar a las obtenidas por García-Batista, et al., (2018) en la validación dominicana, obteniendo para la escala general una consistencia interna óptima $(\alpha=.88)$, siendo las consistencias internas de las subescalas aceptables para la dimensión Cognitiva $(\alpha=.76)$ y Somática $(\alpha=.76)$, aunque baja para la Dimensión Afectiva $(\alpha=.54)$.

\section{Procedimiento}

Se aplicó un diseño de transversal, de caso-control (Woodward, 2014). A partir de la selección inicial de una muestra de personas drogodependientes (casos), se seleccionaron personas de la población general equilibrados en base a las variables sociodemográficas (controles). El instrumento fue aplicado por un miembro del equipo de investigación entrenado en la aplicación y corrección de la escala BDI-II, después de que los participantes firmaran su consentimiento informado. En este estudio se tuvieron en cuenta los lineamientos éticos internacionales al realizar estudios con seres humanos (American Psychological Association, 2017). Al momento de entregar los cuestionarios de autoinforme, todos los participantes fueron debidamente informados de los objetivos de la investigación, el anonimato de sus respuestas, y su participación voluntaria. Asimismo, se aclaró que la participación en la misma no acarrearía ningún daño ni perjuicio para su persona, y que podrían dejar el estudio en cuanto lo deseasen. La presente investigación cuenta con la aprobación del Comité Nacional de Bioética (CONABIOS) perteneciente al Ministerio de Salud Pública de Santo Domingo, República Dominicana (protocolo número 028-2014).

\section{Análisis estadísticos}

En primer lugar, se llevaron a cabo análisis exploratorios iniciales para verificar la normalidad de las pun- tuaciones. Se tuvieron en cuenta las medidas descriptivas de asimetría y curtosis, y se aplicó la Prueba de Normalidad de Kolmogorov-Smirnov (Tabachnick y Fidell, 2007).

Por otra parte, dadas las diferencias observadas entre los grupos respecto a las variables de estado civil y nivel educativo, se decidió aplicar un análisis previo de ANCOVA y MANCOVA. Este análisis permite ingresar covariables al modelo, y de este modo verificar el impacto de las mismas. Asimismo, puede identificarse el efecto que tiene la variable grupo, una vez que se controla el efecto de estas variables sociodemográficas (Hair, Black, Babin, Anderson, y Tatham, 2006).

Luego de evaluar el efecto de las variables sociodemográficas, con el fin de comparar los resultados obtenidos por el grupo clínico y de población general en las puntuaciones del BDI-II, se empleó la prueba $t$ de Student para muestras independientes. En este caso se verificó además el supuesto de homogeneidad de varianza mediante la prueba de Levene. En los casos en donde se verificó el incumplimiento del supuesto (i.e. varianzas desiguales) se procedió a aplicar la prueba t ajustando los grados de libertad mediante el método Welch-Satterthwaite, siendo el procedimiento sugerido para estas situaciones (Zimmerman, 1998). Aquí también se utilizó el estadístico $d$ de Cohen para evaluar el tamaño del efecto. Lo anterior resulta relevante dado que permite no solo basar las conclusiones e implicancias de una investigación en la significación estadística sino también considerando la significación práctica (i.e. magnitud del efecto) de los resultados (Lakens, 2013).

En última instancia, con el objetivo de verificar la eficacia diagnóstica del BDI-II, esto es, la precisión de las puntuaciones del BDI-II para discriminar entre población general y drogodependientes, se aplicó el análisis de las curvas características operativas del receptor (curvas COR). El análisis de curvas COR ha demostrado ser una herramienta estadística óptima para verificar objetivos de este tipo. Para este análisis se calculó las áreas bajo la curva y sus respectivos errores típicos, pruebas de hipótesis e intervalos de confianza (Gönen, 2007). Todos los análisis fueron efectuados con el software estadístico IBM SPSS 20, y la hoja de cálculo de Excel para el obtener el tamaño del efecto desarrollada por Thalheimer y Cook (2002).

\section{Resultados}

\section{Análisis exploratorio inicial}

El análisis inicial de los datos se muestra en la Tabla 3 , en donde se observa una distribución inicial alejada de 
la normal en el grupo de población general. Siguiendo el criterio de detección y eliminación de atípicos univariados (Tabachnick y Fidell, 2007) se procedió a eliminar cuatro casos, luego de lo cual puede apreciarse que el grupo de población general no incumple el supuesto de distribución normal (Tabla 4).

Tabla 3. Análisis descriptivos de las puntuaciones del BDI-II entre población general $(\eta=57)$ y drogodependiente $(\eta=54)$

\begin{tabular}{lcc}
\hline & \multicolumn{2}{c}{ Depresión general } \\
\cline { 2 - 3 } & Población general & Drogodependientes \\
\hline Media & 6.85 & 18.48 \\
DT & 6.50 & 9.29 \\
$g 1$ & 1.62 & 0.43 \\
$g 2$ & 2.89 & 0.04 \\
Kolmogorov-Smirnov & $0.19 * *$ & 0.08 \\
\hline
\end{tabular}

Nota. $* * p<.01, g 1=$ asimetría, $g 2=$ curtosis.

Tabla 4. Análisis descriptivos de las puntuaciones del BDI-II entre población general $(n=53)$ y drogodependiente $(n=54)$

\begin{tabular}{lcc}
\hline & \multicolumn{2}{c}{ Depresión general } \\
\cline { 2 - 3 } & Población general & Drogodependientes \\
\hline Media & 5.56 & 18.48 \\
$D T$ & 4.47 & 9.29 \\
$g 1$ & 0.99 & 0.43 \\
$g 2$ & 0.62 & 0.04 \\
Kolmogorov-Smirnov & 0.11 & 0.08 \\
\hline
\end{tabular}

Nota: $g 1=$ asimetría, $g 2=$ curtosis.

Dado que la eliminación de estos casos pudo tener algún cambio en la distribución de las variables de sexo y edad, se constató nuevamente que las mismas sigan equilibradas en los grupos. Pudo verificarse la equivalencia en los grupos en términos de sexo (\% varones población general $=83.3, \%$ varones drogodependientes $=$ $84.5, \chi^{2}=.03, p=.87$ ) y edad ( $M$ población general $=$ $34.19, M$ drogodependientes $=34.48, t=-.13, p=.90)$.

Análisis de covarianzas univariado y multivariado:

Efecto de la pertenencia a los grupos y de las

covariables sociodemográficas.

Al efectuar los análisis ANCOVA y MANCOVA fue posible verificar que las variables de estado civil y nivel de estudios no tienen un efecto significativo sobre la depresión. En efecto, el ANCOVA muestra que la variable grupo es la única que predice de manera estadísticamen- te significativa la depresión general $(F=37.73, p<.01$, $\eta 2=.27)$, no así la variable estado civil $(F=.16, p=.68$, $\eta 2=.01)$ y nivel educativo $(F=1.94, p=.16, \eta 2=.02)$. Lo mismo sucede cuando se contemplan las subdimensiones de la depresión, siendo sólo el efecto del grupo estadísticamente significativo (Cognitivo $F=41.31, p<$ $.01, \eta 2=.29$; Afectivo $F=22.77, p<.01, \eta 2=.19$; Somático $F=16.61, p<.01, \eta 2=.14)$ no así la variable estado civil (Cognitivo $F=.39, p=.53, \eta 2<.01$; Afectivo $F=.15, p=.70, \eta 2<.01 ;$ Somático $F=.01, p=.95$, $\eta 2<.01$ ) y nivel educativo (Cognitivo $F=.02, p=.88$, $\eta 2<.01$; Afectivo $F=1.55, p=.21, \eta 2=.02$; Somático $F=3.61, p=.06, \eta 2=.03)$.

\section{Pruebas de diferencias entre grupos: Sintomatología depresiva a nivel general y por dimensiones.}

A continuación, se presentan los resultados de las comparaciones efectuadas entre el grupo de drogodependientes y población general, tanto para el consumo general de las sustancias como para cada sustancia específica (Tabla 5).

Los análisis efectuados permiten ver que las personas con problemáticas de consumo, ya sea considerándolas de manera general o específica, en base a las distintas sustancias, presentan mayores niveles de depresión cuando se las compara con personas de población general. Estas diferencias son de elevada magnitud en todos los casos, como puede apreciarse en las medidas de tamaño del efecto.

\section{Análisis de curvas COR}

En la Figura 1 se puede visualizar las áreas bajo la curva correspondientes a la depresión general, y la dimensión cognitiva, afectiva y somática. En la Tabla 6 se describen los valores correspondientes a las áreas bajo la curva y sus respectivos errores típicos, pruebas de hipótesis e intervalos de confianza.

Si bien la Tabla 6 permite ver que el puntaje general de depresión es el que permite discriminar con más precisión entre población general y drogodependientes, el área bajo la curva de todas las dimensiones restantes da cuenta de una precisión adecuada para discriminar entre estos grupos (Gönen, 2007).

\section{Discusión}

La presente investigación tuvo como principales objetivos: a) verificar la validez del BDI-II para identificar la comorbilidad depresiva en población de drogodependientes, considerando el consumo problemático de dis- 
Tabla 5. Diferencias en los niveles de depresión, general y por dimensión, considerando el consumo múltiple o el consumo específico de sustancias

\begin{tabular}{|c|c|c|c|c|c|c|c|c|}
\hline & & & $n$ & $M$ & $D T$ & $t$ & $p$ & $d$ \\
\hline \multicolumn{9}{|c|}{ Consumo general } \\
\hline \multirow{2}{*}{\multicolumn{2}{|c|}{ Depresión }} & Población general & 53 & 5.57 & 4.47 & \multirow{2}{*}{-9.18} & \multirow{2}{*}{$<.01$} & \multirow{2}{*}{1.78} \\
\hline & & Drogodependientes & 54 & 18.48 & 9.29 & & & \\
\hline \multirow{2}{*}{\multicolumn{2}{|c|}{ Cognitivo }} & Población general & 54 & 1.81 & 1.87 & \multirow{2}{*}{-8.58} & \multirow{2}{*}{$<.01$} & \multirow{2}{*}{1.65} \\
\hline & & Drogodependientes & 55 & 6.98 & 4.04 & & & \\
\hline \multirow{2}{*}{\multicolumn{2}{|c|}{ Afectivo }} & Población general & 54 & 0.85 & 1.37 & \multirow{2}{*}{-6.58} & \multirow{2}{*}{$<.01$} & \multirow{2}{*}{1.25} \\
\hline & & Drogodependientes & 57 & 3.14 & 2.22 & & & \\
\hline \multirow{2}{*}{\multicolumn{2}{|c|}{ Somático }} & Población general & 53 & 3.04 & 2.83 & \multirow{2}{*}{-7.25} & \multirow{2}{*}{$<.01$} & \multirow{2}{*}{1.36} \\
\hline & & Drogodependientes & 58 & 8.38 & 4.76 & & & \\
\hline Marihuan & & & & & & & & \\
\hline & Danmón & Población general & 53 & 5.57 & 4.47 & 605 & & $174+2$ \\
\hline & Depresion & Consume & 36 & 18.56 & 10.58 & -6.95 & $<.01$ & 1.14 \\
\hline & Connitive & Población general & 54 & 1.81 & 1.87 & -606 & $<01$ & 174 \\
\hline & Cognitivo & Consume & 36 & 7.17 & 4.35 & -6.96 & $<.01$ & $1 . / 4$ \\
\hline & & Población general & 54 & 0.85 & 1.37 & & & \\
\hline & Afectivo & Consume & 37 & 3.35 & 2.35 & -5.83 & $<.01$ & 1.38 \\
\hline & & Población general & 53 & 3.04 & 2.83 & & & \\
\hline & Somático & Consume & 37 & 7.97 & 5.20 & -5.25 & $<.01$ & 1.26 \\
\hline Cocaína & & & & & & & & \\
\hline & Denresión & Población general & 53 & 5.57 & 4.47 & -814 & $<01$ & 187 \\
\hline & Depresion & Consume & 41 & 19.32 & 10.07 & -8.14 & $<.01$ & 1.81 \\
\hline & Coonitive & Población general & 54 & 1.81 & 1.87 & -7.43 & $<.01$ & 1.69 \\
\hline & Cognitivo & Consume & 42 & 7.17 & 4.37 & -1.43 & $<.01$ & 1.09 \\
\hline & Afectivo & Población general & 54 & 0.85 & 1.37 & -6.07 & $<01$ & 134 \\
\hline & & Consume & 42 & 3.29 & 2.30 & & . & \\
\hline & Somótico & Población general & 53 & 3.04 & 2.83 & 660 & $<01$ & 145 \\
\hline & Solitalico & Consume & 43 & 8.70 & 5.01 & -0.00 & -.01 & $1.4 \mathrm{~J}$ \\
\hline Crack & & & & & & & & \\
\hline & Denresión & Población general & 53 & 5.57 & 4.47 & -5.18 & $<.01$ & 1.83 \\
\hline & Depresion & Consume & 20 & 16.45 & 8.98 & -5.18 & $<.01$ & 1.83 \\
\hline & & Población general & 54 & 1.81 & 1.87 & & & \\
\hline & Cognitivo & Consume & 20 & 6.25 & 4.04 & -4.72 & $<.01$ & 1.71 \\
\hline & & Población general & 54 & 0.85 & 1.37 & & & \\
\hline & Afectivo & Consume & 20 & 2.65 & 2.41 & -3.15 & $<.01$ & 1.07 \\
\hline & Somótico & Población general & 53 & 3.04 & 2.83 & 433 & $<01$ & 130 \\
\hline & Somatico & Consume & 20 & 7.55 & 4.32 & -4.53 & $<.01$ & 1.39 \\
\hline Alcohol & & & & & & & & \\
\hline & Denresión & Población general & 53 & 5.57 & 4.47 & -709 & $<01$ & 206 \\
\hline & Depresion & Consume & 19 & 20.89 & 9.04 & -1.09 & $<.01$ & \\
\hline & Coonitivo & Población general & 54 & 1.81 & 1.87 & -542 & $<01$ & 212 \\
\hline & Cognitivo & Consume & 19 & 7.79 & 4.67 & -5.42 & $<.01$ & 2.12 \\
\hline & Afectivo & Población general & 54 & 0.85 & 1.37 & -531 & $<01$ & 177 \\
\hline & Afectivo & Consume & 20 & 3.75 & 2.29 & -5.31 & $<.01$ & 1.77 \\
\hline & & Población general & 53 & 3.04 & 2.83 & 640 & & \\
\hline & Somatico & Consume & 20 & 9.15 & 3.90 & -6.40 & $<.01$ & 1.97 \\
\hline Nicotina & & & & & & & & \\
\hline & Denresión & Población general & 53 & 5.57 & 4.47 & -364 & $<01$ & 197 \\
\hline & Depresion & Consume & 12 & 17.58 & 11.22 & -3.64 & $<.01$ & 1.91 \\
\hline & Coonitivo & Población general & 54 & 1.81 & 1.87 & -3.18 & $<.01$ & 180 \\
\hline & cogmitivo & Consume & 12 & 6.75 & 5.29 & & -.01 & \\
\hline & Afectivo & Población general & 54 & 0.85 & 1.37 & $-3,56$ & $<01$ & 161 \\
\hline & Alectivo & Consume & 14 & 3.64 & 2.84 & -5.50 & $<.01$ & 1.01 \\
\hline & & Población general & 53 & 3.04 & 2.83 & & & \\
\hline & Somatico & Consume & 14 & 7.79 & 4.56 & -3.71 & $<.01$ & 1.48 \\
\hline
\end{tabular}


Tabla 6. Análisis de curvas COR: Áreas bajo la curva

\begin{tabular}{|c|c|c|c|c|c|}
\hline & \multicolumn{5}{|c|}{ Áreas bajo la curva } \\
\hline & \multirow[b]{2}{*}{ Área } & \multirow[b]{2}{*}{ Error típico } & \multirow[b]{2}{*}{$p$} & \multicolumn{2}{|c|}{ IC asintótico al 95\% } \\
\hline & & & & Límite inferior & Límite superior \\
\hline Depresión & .90 & .03 & $<.01$ & .85 & .96 \\
\hline Cognitiva & .88 & .03 & $<.01$ & .82 & .94 \\
\hline Afectiva & .84 & .04 & $<.01$ & .76 & .91 \\
\hline Somática & .84 & .04 & $<.01$ & .76 & .91 \\
\hline
\end{tabular}

Nota: IC = intervalo de confianza.

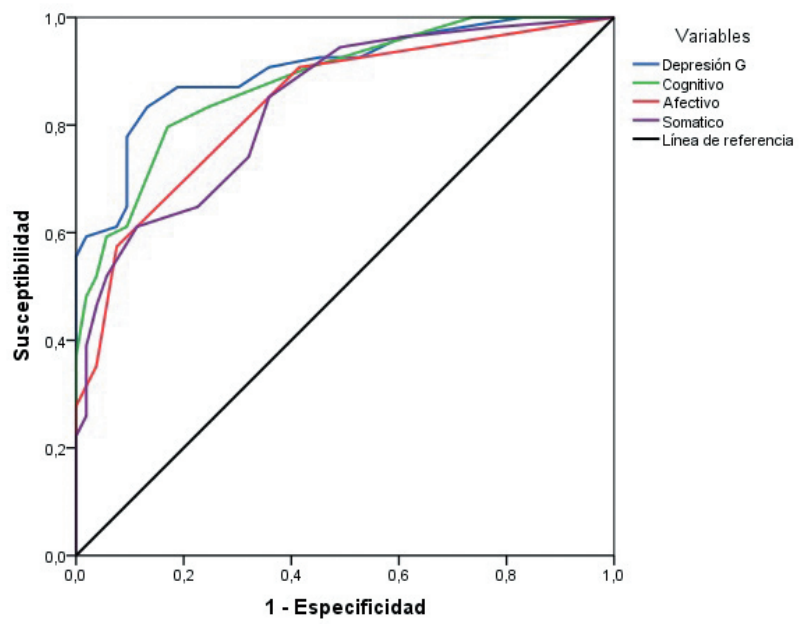

Figura 1. Áreas bajo la curva.

tintas sustancias (i.e. marihuana, cocaína, crack, alcohol, y nicotina); y b) verificar la eficacia diagnóstica, respecto a la distinción entre población general y drogodependientes clínicos, del BDI-II. Para cumplimentar el primer objetivo se llevaron a cabo sucesivos análisis de diferencias entre grupos (i.e. prueba $t$ ), controlando en base a las variables sociodemográficas. Mientras que, para el segundo objetivo, se aplicó un análisis de curvas COR.

Respecto al primer objetivo, en términos generales los resultados indican que los niveles de depresión, tanto en su dimensión general, como en sus componentes cognitivos, afectivos y somáticos, son significativamente mayores en el grupo de personas drogodependientes. Más aún, estas diferencias con respecto a la población general son de gran magnitud, y la sintomatología depresiva evidenciada no muestra tener variaciones en base al consumo de las distintas sustancias. En otras palabras, las puntuaciones del BDI-II han demostrado validez discriminativa más allá del tipo de consumo particular (e.g. marihuana vs cocaína), siendo el nivel de depresión general y específico, según la dimensión considerada, marcadamente mayor en las personas con problemática de consumo. En cuanto al segundo objetivo, el análisis de curvas COR aporta evidencia a favor de la precisión del BDI-II para diferenciar adecuadamente entre población general y drogodependientes. Como se mencionó en la introducción existe escasa evidencia acerca de la aplicabilidad, en términos de validez, del BDI-II en drogodependientes clínicos. Aun así los datos obtenidos en nuestro estudio son coherentes con estos antecedentes, en la medida de que avalan la aplicación de este inventario en personas con problemas de abuso de sustancias (Moore, Neale, Silberg, y Verhulst, 2016; Seignourel, et al., 2008).

Sin embargo, es preciso remarcar que la validez en cuanto a herramienta de screening en población de drogodependientes que se ha obtenido en la presente investigación es parcial. En nuestro estudio el tamaño muestral no permitió verificar en primera instancia el modelo de medida (Byrne, 2010), esto es, la replicabilidad de las propiedades psicométricas, de validez y confiabilidad, del BDI-II en drogodependientes de República Dominicana. Es de destacar en este sentido que la subdimensión Afectiva del inventario no obtuvo una consistencia interna adecuada en la muestra objeto de estudio, lo cual puede estar sugiriendo una estructura factorial diferente en esta población clínica. Por lo cual, dados los resultados obtenidos en la validación dominicana del instrumento (García-Batista, et al., 2018), y la controversia aún vigente en cuanto a la consideración de subdimensiones en el BDI-II (Brouwer, Meijer, y Zevalkink, 2013; Heinrich, Zagorscak, y Knaevelsrud, 2018), se sugiere aplicar la medida global, de severidad depresiva.

Relacionado con esto último, si bien los datos sugieren además que la validez de la aplicabilidad del BDI-II en personas con problema de abuso de sustancias sería invariante en función de las variables socio-culturales, dadas las particularidades del contexto Dominicano y sus diferencias con las sociedades WEIR (Western, educated, industrialized, and rich; Rad, et al., 2018), se reitera la necesidad de verificar las propiedades psicométricas, particularmente de validez de constructo, en población con problemática de consumo. Futuras inves- 
tigaciones deberán corroborar dichas propiedades, así como su invarianza factorial en base al consumo, el género y la edad (Skule, et al., 2014). Debe también tenerse presente que, dadas las limitaciones de tiempo y espacio, en el presente trabajo no pudieron medirse algunas variables de relevancia que deberían contemplarse en futuros estudios, como ser la presencia en los participantes de otros trastornos psiquiátricos y el consumo de medicamentos psicotrópicos (Seignourel, et al., 2008).

Contar con instrumentos de medición válidos y confiables, adaptados al contexto socio-cultural, es la base fundamental a partir de lo cual puede lograrse un correcto avance en las áreas aplicadas y de investigación. Los resultados obtenidos permiten ampliar la evidencia favorable respecto a la validez del BDI-II como instrumento de screening para ser aplicado en personas con drogodependencia.

\section{Conflictos de intereses}

Los autores declaran que no existen conflictos de intereses.

\section{Referencias}

American Psychiatric Association (2000). Diagnostic and statistical manual of mental disorders. (4th ed., text revision). Washington, DC: American Psychiatric Association.

American Psychological Association (2017). Ethical Principles of Psychologists and Code of Conduct. Washington, DC: Author. Retrieved from http://www.apa.org/ethics

Beaulieu, T., Ti, L., Milloy, M., Nosova, E., Wood, E., y Hayashi, K. (2018). Major depressive disorder and access to health services among people who use illicit drugs in Vancouver, Canada. Substance Abuse Treatment, Prevention, And Policy, 13(1). doi: 10.1186/s13011-018-0142-9

Beck, A., Steer, R., y Brown, G. (1996). Beck Depression Inventory (second edition) [Inventario de depresión de Beck (segunda edición)]. San Antonio, Texas: Psycho-logical Corporation.

Brouwer, D., Meijer, R. R., y Zevalkink, J. (2013). On the factor structure of the Beck Depression Inventory-II: G is the key. Psychological Assessment, 25(1), 136-145. doi:10.1037/ a0029228

Byrne, B. M. (2010). Structural equation modeling with AMOS. Basic concepts, applications, and programming. New York, NY: Routledge.

Crnić, K. B., Todorović, M. M., Marković, S. Z., Kastratović, D. A., y Timotijević, I. P. (2016). Addiction and depression comorbidity approaches. Hospital Pharmacology: International Multidisciplinary Journal, 3(3), 440-446. doi: 10.5937/hpimj1603440C

Gajewski, P. A., Turecki, G., y Robison, A. J. (2016). Differential expression of FosB proteins and potential target genes in select brain regions of addiction and depression patients. PLoS One, 11(8), 1-14. doi: 10.1371/journal.pone.0160355
García-Batista, Z. E., Guerra-Peña, K., Cano-Vindel, A., HerreraMartínez, S.X., y Medrano, L. A. (2018). Validity and reliability of the Beck Depression Inventory (BDI-II) in general and hospital population of Dominican Republic. PLoS One, 13(6), e0199750. doi: 10.1371/journal.pone.0199750

García-García, V., Luque, B., Santos Ruiz, M., y Tabernero, C. (2017). La autorregulación emocional en la depresión y el deterioro cognitivo de consumidores de sustancias psicoactivas. Health y Addictions/Salud y Drogas, 17(2), 125-136.

Gönen, M. (2007). Analyzing Receiver Operating Characteristic Curves with SAS. New York, NY: SAS Publishing.

Gómez-Coronado, N., Sethi, R., Bortolasci, C. C., Arancini, L., Berk, M., y Dodd, S. (2018). A review of the neurobiological underpinning of comorbid substance use and mood disorders. Journal of Affective Disorders, 241, 388-401. doi: 10.1016/j. jad.2018.08.041

Hair, J. F., Black, W. C., Babin, B. J., Anderson, R. E., y Tatham, R. L. (2006). Multivariate data analysis. New York, NY: Uppersaddle River.

Hammond, E. R., Lai, S., Wright, C. M., y Treisman, G. J. (2016). Cocaine use may be associated with increased depression in persons infected with HIV. AIDS and Behavior, 20(2), 345352. doi: 10.1007/s10461-015-1187-7

Heinrich, M., Zagorscak, P., Eid, M., y Knaevelsrud, C. (2018). Giving G a Meaning: An Application of the Bifactor-(S-1) Approach to Realize a More Symptom-Oriented Modeling of the Beck Depression Inventory-II. Assessment, 1-19. doi: $10.1177 / 1073191118803738$

Hodgson, K., Almasy, L., Knowles, E. M., Kent, J. J., Curran, J. E., Dyer, T. D.,... Glahn, D. C. (2017). The genetic basis of the comorbidity between cannabis use and major depression. Addiction, 112(1), 113-123. doi: 10.1111/add.13558

Johnson, M. E., Neal, D. B., Brems, C., y Fisher, D. G. (2006). Depression as measured by the Beck Depression Inventory-II among injecting drug users. Assessment, 13(2), 168-177. doi: $10.1177 / 1073191106286951$

Kennett, J., Matthews, S., y Snoek, A. (2013). Pleasure and addiction. Frontiers in Psychiatry, 4. doi: 10.3389/fpsyt.2013. 00117

Khalid, S., y Kausar, R. (2016). Protective factors, drug use and depression in young drug users. Journal of Behavioural Sciences, 26(2), 116-134.

Kohn, R., Ali, A. A., Puac-Polanco, V., Figueroa, C., López-Soto, V., Morgan, K., ... Vicente, B. (2018). Mental health in the Americas: an overview of the treatment gap. Revista Panamericana de Salud Pública, 42. doi:10.26633/rpsp.2018.165

Lai, H. M. X., Cleary, M., Sitharthan, T., y Hunt, G. E. (2015). Prevalence of comorbid substance use, anxiety and mood disorders in epidemiological surveys, 1990-2014: A systematic review and meta-analysis. Drug and Alcohol Dependence, 154, 1-13.

Lakens, D. (2013). Calculating and reporting effect sizes to facilitate cumulative science: a practical primer for t-tests and ANOVAs. Frontiers in Psychology, 4. doi:10.3389/fpsyg.2013. 00863

Lister, J. J., Ledgerwood, D. M., Lundahl, L. H., y Greenwald, M. K. (2015). Causal pathways between impulsiveness, cocaine use consequences, and depression. Addictive Behaviors, 41, 411-416. doi: 10.1016/j.addbeh.2014.09.017 
Moore, A. A., Neale, M. C., Silberg, J. L., y Verhulst, B. (2016) Substance use and depression symptomatology: Measurement invariance of the Beck Depression Inventory (BDI-II) among non-users and frequent-users of alcohol, nicotine and cannabis. Plos ONE, 11(4), 1-13. doi: 10.1371/journal.pone.0152118

Obasi, E. M., Brooks, J. J., y Cavanagh, M. (2016). The relationship between psychological distress, negative cognitions, and expectancies on problem drinking: exploring a growing problem among university students. Behavior Modification, 40(1-2), 51-69. doi: 10.1177/0145445515601793

Organización Mundial de la Salud. (2018). Depresión [Nota descriptiva]. Recuperado de http://www.who.int/es/newsroom/fact-sheets/detail/depression

Pordanjani, S. R., Zadeh, H. F., Mousavi, M., Khazaei, S., Sohrabivafa, M., Momenabadi, V.,...Khazaei, Z. (2018). Prevalence and reasons for psychoactive drugs use among university students of Medical Sciences in Yazd, Iran. Iranian Journal of Psychiatry and Behavioral Sciences, 12(1). doi: 10.5812/ijpbs.9384

Rao, U. (2006). Links between depression and substance abuse in adolescents: Neurobiological mechanisms. American Journal of Preventive Medicine, 31(6S1), 161-174. doi: 10.1016/j. amepre.2006.07.002

Rad, M. S., Martingano, A. J., y Ginges, J. (2018). Toward a psychology of Homo sapiens: Making psychological science more representative of the human population. Proceedings of the National Academy of Sciences, 115(45), 11401-11405. doi:10.1073/pnas.1721165115

Sanz, J., Gutiérrez, S., Gesteira, C., y García-Vera, M. P. (2014). Criterios y baremos para interpretar el «Inventario de Depresión de Beck-II»(BDI-II). Behavioral Psychology, 22, 37-59.

Seignourel, P. J., Green, C., y Schmitz, J. M. (2008). Factor structure and diagnostic efficiency of the BDI-II in treatmentseeking substance users. Drug and Alcohol Dependence, 93(3), 271-278. doi: 10.1016/j.drugalcdep.2007.10.016
Selby, E. A., Anestis, M. D., y Joiner, T. E. (2008). Understanding the relationship between emotional and behavioral dysregulation: Emotional cascades. Behaviour Research and Therapy, 46(5), 593-611. doi: 10.1016/j.brat.2008.02.002

Selby, E. A., Franklin, J., Carson-Wong, A., y Rizvi, S. L. (2013). Emotional Cascades and Self-Injury: Investigating Instability of Rumination and Negative Emotion. Journal of Clinical Psychology, 69(12), 1213-1227. doi: 10.1002/jclp.21966

Skule, C., Ulleberg, P., Dallavara Lending, H., Berge, T., Egeland, J., Brennen, T., y Landrø, N. I. (2014). Depressive symptoms in people with and without alcohol abuse: factor structure and measurement invariance of the Beck Depression Inventory (BDI-II) across groups. PLoS One, 9(2), e88321. doi: 10.1371/ journal.pone.0088321

Sloan, E., Hall, K., Moulding, R., Bryce, S., Mildred, H., y Staiger, P. K. (2017). Emotion regulation as a transdiagnostic treatment construct across anxiety, depression, substance, eating and borderline personality disorders: A systematic review. Clinical Psychology Review, 57, 141-163. doi: 10. 1016/j.cpr.2017.09.002

Tabachnick, B. G., y Fidell, L. S. (2007). Using Multivariate Statistics. New York, NY: Pearson Education.

Thalheimer, W., y Cook, S. (2002). How to calculate effect sizes from published research: A simplified methodology. WorkLearning Research, 1. Recuperado de www.work-learning. com/effect_sizes.htm.

Webb, E., Ashton, C. H., Kelly, P. y Kamali, F. (1996). Alcohol and drug use in UK university students. The Lancet, 348(9032), 922-925. doi:10.1016/S0140-6736(96)03410-1

Woodward, M. (2014). Epidemiology. Study Design and Data Analysis. New York, NY: CRC Press.

Zimmerman, D. W. (1998). Invalidation of Parametric and Nonparametric Statistical Tests by Concurrent Violation of Two Assumptions. The Journal of Experimental Education, 67(1), 55-68. doi:10.1080/00220979809598344 
